\title{
Urgent Percutaneous Coronary Artery Intervention and Coronary Artery Bypass Grafting in STEMI Patients with Confirmed COVID-19
}

\author{
Gökhan Keskin, ${ }^{1}$ Ayhan Uysal, MD,${ }^{2}$ Esra Erturk, MD,${ }^{3}$ Erhan Hafiz, MD,${ }^{4}$ Omer Faruk Dogan, $M D^{5}$ \\ ${ }^{1}$ Department of Cardiology, Amasya University School of Medicine, Research and Education Hospital, Amasya, Turkey; ${ }^{2}$ Department of \\ Cardiovascular Surgery, Firat University School of Medicine, Elazig, Turkey; ${ }^{3}$ Department of Cardiovascular Surgery, Mersin City Hospital, \\ Mersin, Turkey; ${ }^{4}$ Gaziantep University School of Medicine, Gaziantep, Turkey; ${ }^{5}$ Department of Cardiovascular Surgery, Adiyaman University \\ School of Medicine, Research and training Hospital, Adıyaman, Turkey
}

\section{ABSTRACT}

Background: Our aim of this study was to evaluate the cardiac symptoms, coronary angiographic results, and clinical outcomes of patients with confirmed COVID-19 and STsegment elevation with myocardial infarction (STEMI) or myocardial ischemia.

Material and methods: Thirty-seven patients, who already were confirmed with COVID-19 using reverse transcriptase-polymerase chain reaction (RT-PCR), were admitted to our hospital due to chest pain with STEMI. The median patient age was 66 years (range: $27-84$ years). Female/male ratio was $22 / 15$. We performed a second RT-PCR test in all patients. We investigated myocardial enzymes (creatine kinase myocardial band (CK-MB), cardiac troponin-I (c-TnI), and C-reactive protein (CRP), and liver enzymes (alanine amino transferase (ALT) and aspartate amino transferase (AST) also were measured. Blood d-dimer, thromboplastin time (PT), partial thromboplastin time (PTT), and fibrinogen were investigated. Transcutaneous oxygen saturation was monitored for each patient in the emergency department (ED). To evaluate myocardial wall abnormalities, transthoracic echocardiography was performed.

Results: Coronary artery disorders requiring revascularization were detected in 25 patients $(67.5 \%)$. There was no evidence of coronary artery disease in the remaining 12 patients. Out of 25 , nine coronary artery disease patients had a history of coronary intervention (24.3\%). All patients had high levels of myocardial enzyme release. Percutaneous coronary interventions (PCI) were performed in patients with culprit lesion(s). Success rate of PCI was $87.5 \%(\mathrm{~N}=$ 21). The median number of stent use was $2.9 \pm 0.7$ (range: $1-4$ ). Because PCI failed in four patients, we suggested elective coronary artery bypass grafting (CABG) surgery after medi$\mathrm{cal}$ treatment. Six patients required re-intervention owing to early stent thrombosis (30\%). Seven patients died after PCI

Received December 20, 2020; received in revised form March 4, 2021; accepted March 5, 2021.

Correspondence: Dr. Gökhan Keskin, Assistant Prof., Amasya University School of Medicine, Department of Cardiology, No:1/1, Amasya, Turkey; +903582115005; fax: +903582180104 (e-mail: ofdogan@hacettepe.edu.tr).
(33.3\%). For patients with negative or positive RT-PCR test results, we performed thoracic computed tomography (CT), which is a sensitive diagnostic method for COVID-19. Interlobular septal and pleural thickening with patchy bronchiectasis in the bilateral or unilaterally lower and/or middle lobe(s) were the main pathologies in 24 patients. D-dimer, fibrinogen, and CRP levels were high in 11 PCI patients with bilaterally pulmonary involvement by COVID-19 (52.3\%), while fibrin degradation products did not significantly change. For three patients with normal coronary arteries with a transient hypokinesia or hypokinesia as result of myocarditis, we decided to perform atypical Takotsubo cardiomyopathy. We medically treated using inodilator (levosimendan), diuretic, angiotensin-converting enzyme inhibitors and beta-blockers. To prevent the risk of thromboembolism, we also administered a heparin drip. The myocardial contractility of the apex did improve, and patients were discharged from the hospital, with the exception of one young female patient. She is following in the ICU with stabil hemodynamics.

Conclusion: Chest pain with STEMI can develop in patients with confirmed COVID-19. Nearly one-third of patients had COVID-19 with chest pain and concomitant STEMI and normal coronary angiography (32.4\%). Urgent PCI may be performed in hemodynamically unstable patients with high mortality. Complications, including sudden cardiac arrest, severe ventricular arrhythmia, and Takotsubo cardiomyopathy, related to COVID-19 patients with normal coronary arteries.

\section{INTRODUCTION}

The first case with SARS-CoV-2 2019 (COVID-19) was reported in December, in Wuhan City [Chen 2020]. It was declared a global pandemic by the World Health Organization (WHO), affecting more than 200 countries [World Health Organization 2019]. Dyspnea, dry cough, loss of smell, and sore throat are the main symptoms of COVID19. Chest pain with STEMI as strong typical myocardial infarction have been reported in rare patients confirmed with COVID-19 [Welt 2020; Ing 2020; Curzen 2020]. Studies also have shown that myocardial injury related to cytokine storm may be seen during the course of COVID-19 in patients with risk factors, such as hypertension or diabetes mellitus [Chieffo 2020; Bangalore 2020; Giulio 2020; Shi 2020; Huang 2020]. 
Table 1. Patient characteristics

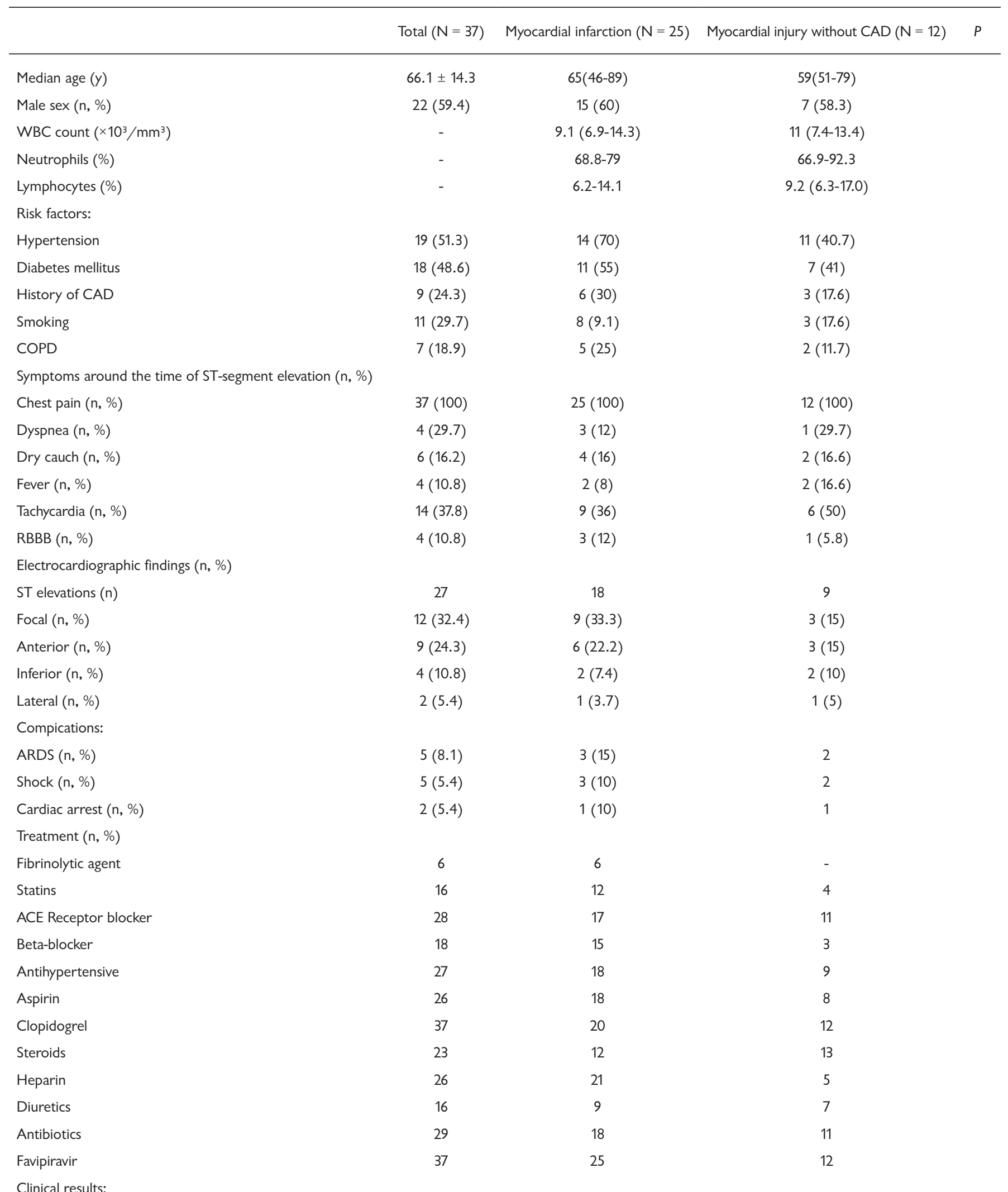


Table 1. Patient characteristics [CONT.]

\begin{tabular}{|c|c|c|c|c|}
\hline Mortality & $9(24.3)$ & $6(24)$ & $3(25)$ & .87 \\
\hline Normal EF & $23(59.4)$ & $17(33.3)$ & $6(70)$ & .020 \\
\hline Low EF $(\leq 40)$ & $18(40.5)$ & $10(40.7)$ & $8(20)$ & .034 \\
\hline Reintervention (n, \%) & $6(16.2)$ & $6(14.8)$ & - & \\
\hline
\end{tabular}

WBC, white blood cell; CAD, coronary artery disease; ACE, angiotensin converting enzyme; TTE, transthoracic echocardiography; EF, ejection fraction; COPD, chronic obstructive pulmonary disease

In recent studies, the authors described Takotsubo syndrome (TS) in an older female patient confirmed with COVID-19 [Kariyanna 2020; Singh 2020; Bangalore 2020; Dabbagh 2020; Meyer 2020]. The first case of an apical variant of Takotsubo cardiomyopathy (T-CMP) because of COVID-19 recently was reported by Kariyanna et al as a new entity [Kariyanna 2020]. The first review of T-CMP in 12 COVID-19 patients recently was reported by Shing et al [Singh 2020]. In this review, Singh et al argued that the cytokine storm that occurs during COVID-19 infection can cause TS by triggering events, such as when chemicals release during emotional stress, which may precipitate TS [Bangalore 2020; Dabbagh 2020]. In these rare patients, ventriculography or TTE showed the apical ballooning in most patients [Meyer 2020], apical hypokinesia or akinesia with or without basal hyperkinesia, and basal or mild hypokinesia characteristic of TS variants. Most patients with TS recovered and were discharged after classical medical treatment. However, the authors have reported severe complications, such as cardiac tamponade, cardiogenic shock, myocarditis, hypertensive crisis, or respiratory failure in more than nine patients confirmed with COVID-19. Unfortunately, we are facing unusual and amazing situations related to COVID-19.

Urgent revascularization in COVID-19 patients with CAD were admitted to the hospital because of acute myocardial infarction that brought some challenges to cardiologists and professional healthcare workers (PHWs), during the pandemic [Bangalore 2020; Giulio 2020; Shi 2020; Huang 2020; Kariyanna 2020; Singh 2020; Bangalore 2020]. Thus, the American College of Cardiology and Society of Cardiovascular Angiography and Intervention discussed how to manage STEMI for the safety of patients and PHWs, during the COVID-19 pandemic [Welt 2020].

To prevent in-hospital nasocomial infection during myocardial revascularization, Ing et al proposed thrombolysis as the first treatment strategy in hemodynamically stable COVID-19 patients with STEMI with confirmed CAD [Ing 2020]. In contrast, some clinicians suggested proceeding with the primary PCI during the pandemic period as an elective situation [Sala 2020; Zhou 2020; Guo 2020; AlGhatrif 2020;
Choudry 2020; Xuan 2020; World Health Organization 2005]. Shi et al showed that patients with cardiac injury had higher mortality than those without cardiac injury [51.2\% versus $4.5 \%$ ] [Giulio 2020]. We should keep in mind that inhospital COVID-19 spread may increase from admission to discharge from the hospital. Thus, there is no common consensus yet for the management of COVID-19 patients who have STEMI with severe CAD. On the other hand, there are only a few scientific reports about the coronary angiography results in COVID-19 patients with STEMI and chest pain [Bangalore 2020; Giulio 2020; Shi 2020; Zhou 2020; Guo 2020; AlGhatrif 2020].

The authors stated that only half of the COVID-19 patients with STEMI and chest pain had severe CAD, which was confirmed with coronary angiography [Curzen 2020; Chieffo 2020; Bangalore 2020; Giulio 2020; Shi 2020; Huang 2020; Kariyanna 2020; Singh 2020; Bangalore 2020; Dabbagh 2020; Meyer 2020]. Another important issue is that the cytokine storm related to COVID-19 may be the cause of myocardial injury related to intracoronary microthrombus. Recent studies demonstrated that nearly half of the patients confirmed with COVID-19 presenting with chest pain and STEMI had obstructive CAD [Bangalore 2020; Giulio 2020; Shi 2020; Huang 2020; Kariyanna 2020; Singh 2020; Bangalore 2020; Dabbagh 2020; Meyer 2020; Sala 2020; Zhou 2020; Guo 2020; AlGhatrif 2020; Choudry 2020; Xuan 2020]. Delaying coronary revascularization in CAD patients may increase the risk of severe myocardial infarction because of the restriction of patients' daily activities. A number of CAD patients had systemic hypertension and/or diabetes mellitus. The risk of sudden cardiac arrest, due to severe ventricular arrhytmia resulting from myocardial infarction and/or ischemic cardiomyopathy, are important complications of COVID-19 [Shi 2020; Huang 2020; Kariyanna 2020; Singh 2020; Bangalore 2020].

Here, we discuss the coronary angiography results of STEMI patients confirmed with COVID-19 who had STEMI because of chest pain, and the results of urgent PCI and CABG. We also present the risk/benefit ratio of PCI and CABG in a limited case series confirmed with COVID-19. 


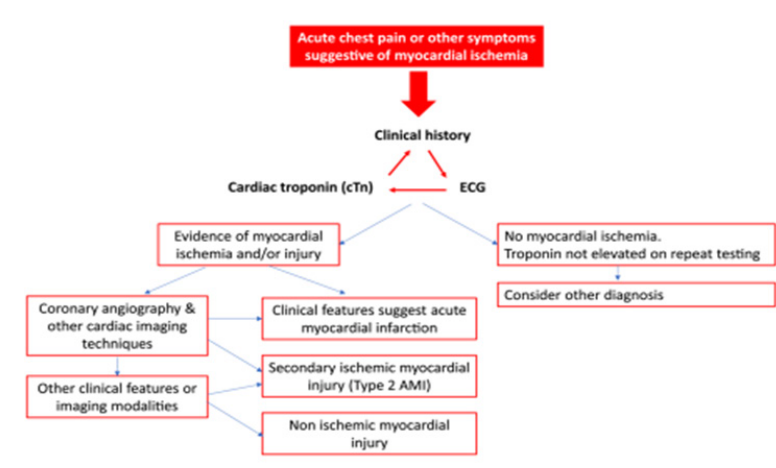

Figure 1. Our algorithm of percutaneous coronary intervention. According to our protocols, we classified the patients requiring coronary artery bypass grafting.

\section{PATIENTS AND METHODS}

We performed coronary angiography in 37 patients with confirmed COVID-19 between March 30, 2020 and December 1, 2020, because they were admitted to the hospital with chest pain in combination with STEMI. All patients received a loading dose of clopidogrel $(600 \mathrm{mg})$ before the coronary angiography. Median age of the patients was $66.1 \pm$ 14.3 years (range: $27-84$ years). Female/male ratio was 22/15. The characteristics of patients, symptoms, medication, blood analyses, enzyme levels, and complications have been summarized (Table 1). The median time from the diagnosis of COVID-19 to hospitalization was $7.4 \pm 3.2$ days (range: $2-10$ days). Fourteen patients had some respiratory symptoms, such as fever, dyspnea, and dry cough (16.2\%). The diagnosis of COVID-19 was made incidentally in eight patients without any symptoms and whose medical treatment started at home while they were under quarantine. STEMI was described according to typical symptoms associated with ST-segment elevation. Coronary artery stenosis was defined as the culprit lesion in patients with the evidence of luminal occlusion or intracoronary thrombus. We described obstructive coronary artery based on the angiographic evidence of a stenosis $>50 \%$. Myocardial injury was defined according to echocardiographic results and blood levels of cardiac biomarkers above the upper reference limits of c-TnI and CK-MB.

Patients with STEMI were triaged in the ED, and a full history (questionnaire) relating to COVID-19 signs and symptoms was taken. RT-PCR tests immediately were performed for each patient after admission to the hospital's ED. Patients with COVID-19 symptoms had positive test results $(\mathrm{N}=14$ patients). Myocardial enzyme release, cardiac troponin-I, and CK-MB regularly were measured. Blood levels of cardiac troponin-I and CK-MB were significantly high in all patients. However, release of enzymes, including c-Tn-I and CK-MB, were higher in patients with CAD than those with normal coronary arteries. The levels of c-Tn-I in patients, who underwent PCI because of myocardial infarction and in patients with myocardial ischemia (normal coronary arteries), were $112(80-440 \mathrm{ng} / \mathrm{mL})$ and

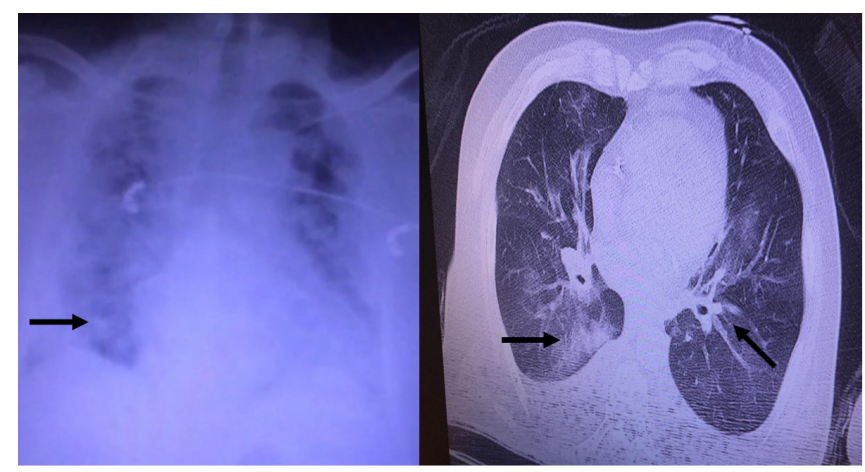

Figure 2. A) Chest x-ray demonstrating bilateral pulmonary consolidation. B) Thoracic computed tomography showing reticular and patchy opacities bilaterally with lung consolidation and pleural thickening, including in the brochyectatic areas, in a male with coronary artery disorders who had COVID-19.

$13.5(4.8-41 \mathrm{ng} / \mathrm{mL})$, respectively $(P=.001)$. CK-MB levels in patients requiring PCI and in those with myocarditis were $84.2(54.2-192.8 \mathrm{U} / \mathrm{L})$ and $64(23-54.7 \mathrm{U} / \mathrm{L})$, respectively $(P=.024)$.

Fibrinogen levels were significantly high in patients who had myocardial infarction (the median level: $1280 \pm 1190 \mathrm{mg} /$ dL) (range: 950-2580 ng/dl). When we compared median level of fibrinogen in PCI patients and the patients with normal coronary arteries, there was a significant difference $(P=.0002)$. The median level of blood $\mathrm{d}$-dimer in the PCI group was statistically significant $(P=.0001)$.

Blood thrombocyte levels, thromboplastin time (TT), activated partial thromboplastin time (aPTT), and international normalized ratio (INR) also were regularly measured in both groups and noted. All measurements were similar in both groups.

All necessary precautions were taken to prevent the spread of COVID-19 to PHWs through nasocomial contamination, considering previous recommendations. To prevent nasocomial spread, we used essential rules and planned the works of professional health care workers step by step for each intervention. According to the American Heart Society, we prepared Cath. Lab. equipment prior to procedures. We used double surgical gloves, face masks (N-95), and face shields. The same precautions were taken for the operating room in CABG operations. We continued the medical treatment of COVID-19 in the hospital, according to infectious disease specialists. We followed the patients using invasive cardiac monitorization. Electrocardiographic records, cardiac enzyme levels, and oxygen saturation were recorded. TTE was done for investigation of myocardial performance.

\section{RESULTS}

According to in-hospital patient management and triage rules, we performed coronary angiography and stenting (Figure 1). Radiologic findings, including chest $\mathrm{x}$-ray and thoracic CT, cardiac enzyme levels, and the coronary artery 
Table 2. Radiologic findings, cardiac enzyme levels and coronary artery lesions of the patients

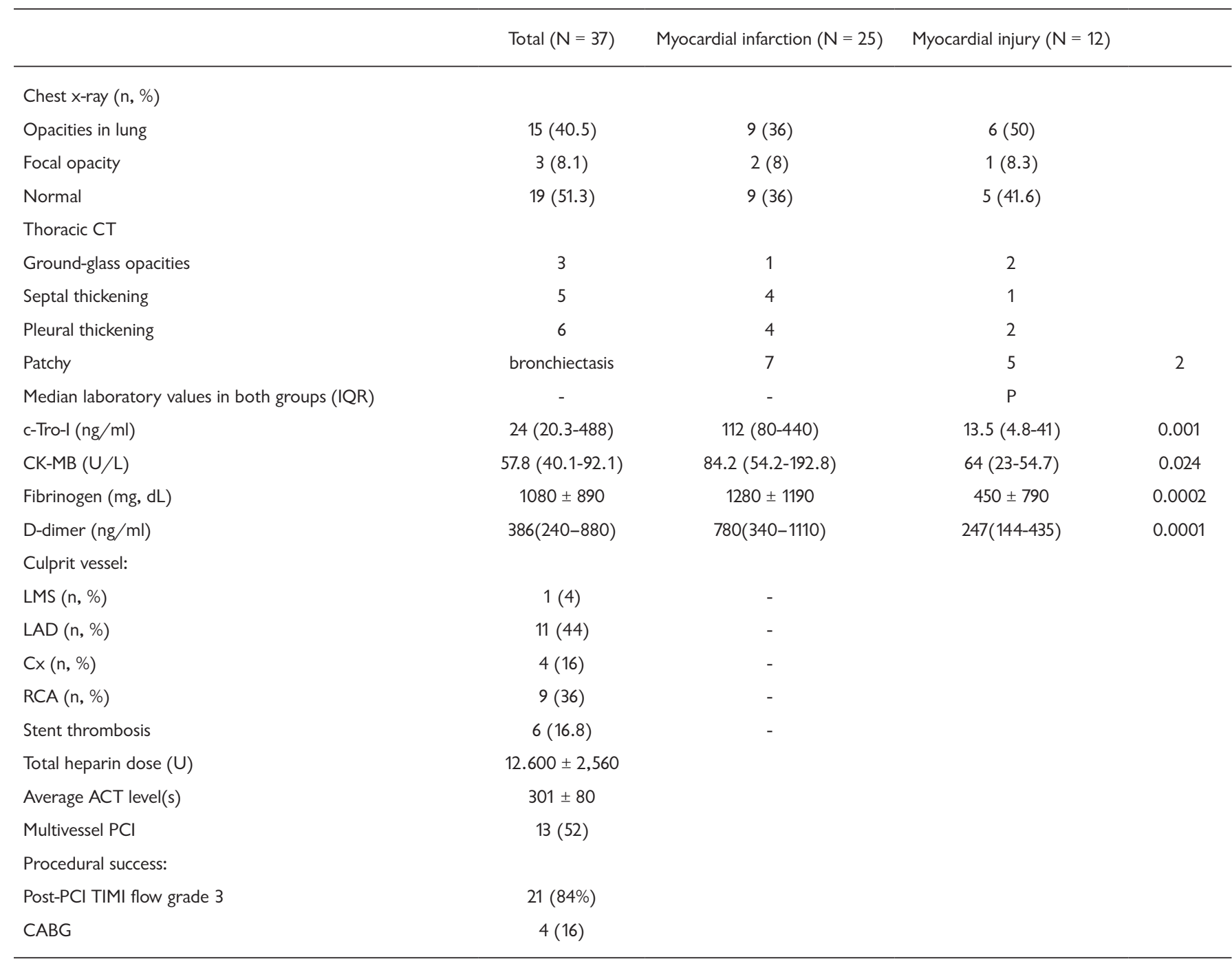

*Because we have a limited number of patients, percentage is not total 100. The value for the troponin I level was 0.01 nanogram/milliliter or less. RBBB, right bundle branch block; CT, computed tomography

lesions were summarized (Table 2). Angiographic results showed that there were coronary artery occlusive diseases in 25 patients $(62.5 \%)$. We detected one or multiple culprit lesions. The number of preexisting coronary artery disease patients with COVID-19 who underwent reintervention was nine (21\%). We determined chronic total occlusion (CTO) of the right coronary artery in five patients. CTO of left anterior descending (LAD) artery was observed in two patients and in the circumflex artery $(\mathrm{Cx})$ in one patient. We performed PCI using DES in 21 patients. The median number of stent use was $2.9 \pm 0.7$ (range:1-4). After succesful PCI, new electrocardiographic abnormalities, such as tachycardia-bradicardia or a new right bundle branch block (RBBB), were seen in five patients. Because PCI failed in four patients who had long segment stenosis or chronic total occlusion of LAD artery, we recommended CABG surgery as an elective situation after medical treatment in hemodynamically stable patients. We isolated all patients in separate rooms until surgery. Blood analyses, fibronogen levels, and coagulation factors (INR, PT, APTT) regularly were measured. Thoracic CT was performed prior to CABG for each patient. Oxygen saturation in CABG patients was more than $90-95 \%$ on room air. All CABG surgeries were performed using cardiopulmonary bypass one week following medical treatment.

On echocardiography, 24 patients had localized wall motion abnormalities, apical or global hypokinesia or akinesia. Out of 24 , nine patients had normal coronary arteries. We intravenously started high dose methylprednisolone administration in combination with inotropics in patients with normal coronary angiography. Because three patients with normal coronary arteries had transient hypokinesia or akinesia as a result of myocarditis, we decided to perform an atypical T-CMP. These 


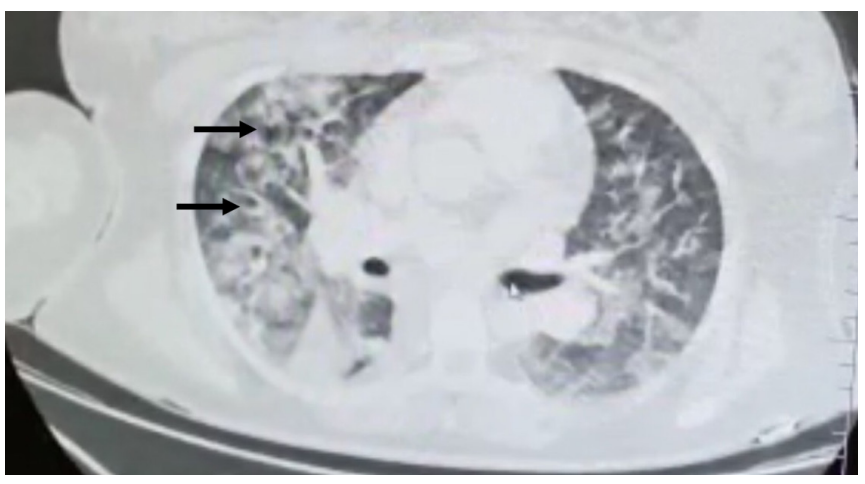

Figure 3. Axial section of thoracic computed tomography demonstrates the pulmonary infiltration and bilateral subpleural ground-glass opacities as typical findings of COVID-19 pneumonia in patients with clinical presentation for COVID-19 infection. This male patient presented with fever and cough. Here, bilateral lung consolidation combined with edema and pleural thickening are observed.

patients had apical akinesia/hypokinesia with ST-segment elevation. All evidence showed "broken heart syndrome" or Takotsubo syndrome. We started medical treatment with an inodilator agent (levosimendan), high dose intravenous steroid administration, diuretic, angiotensin-converting enzyme inhibitor and beta-blockers. To prevent the risk of thromboembolism, we also administered a heparin drip. The myocardial contractility of the apex improved after 10 days of treatment, and these patients were discharged from hospital, except one young female patient. Unfortunately, a major cerebral embolic event developed during ICU follow up, and she died due to multiorgan failure on the 17 th postoperative day.

Preexisting CAD, diabetes mellitus and hypertension were the most prevalant risk factors in patients who required PCI (72\% of patients). In patients without coronary artery disorders $(\mathrm{N}=12)$, we detected diabetes mellitus in nine $(75 \%)$. There were no significant differences in the groups, when comparing risk factors $(P=.86)$. Global myocardial hypokinesia or wall abnormalities were the most common findings of TTE in patients with CAD who underwent PCI (72\% versus $58.3 \%)(P=.0022)$.

Hemodynamic instability was observed in six patients $(28.5 \%)$ with multiple coronary artery disease with low left ventricular ejection fraction. At the end of first day after successful PCI, sudden cardiac arrest developed in two patients. Cardiopulmonary resuscitation was performed, and fibrinolytic agent administration immediately was started. An urgent angiography was performed for stent control. In these two patients, there was instant restenosis in the LAD and RCA artery. We performed reinterventions together with fibrinolytic agent and continuous heparin administration. We started Glicoprotein IIA-IIIB as an anticoagulant. The blood levels of d-dimer, fibrinogen, cardiac troponin-I, and CK-MB levels increased in these two patients. During the follow-up period, one patient died due to multiorgan failure.

We routinely performed chest x-ray in all patients after PCI. In seven patients, the median level of blood oxygen saturation was $78 \pm 14 \%$ in room air (range: $76-96 \%$ ). Chest
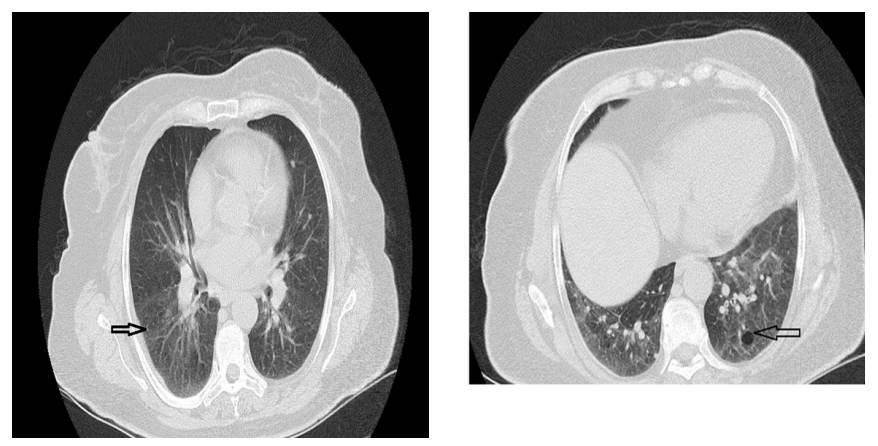

Figure 4. Thoracic computed tomography demonstrates the pulmonary infiltration and bilateral subpleural ground-glass opacities (white arrow) in a hypertension and diabetic female patient. There are subpleural ground-glass opacities and bronchyectasia (right side, arrowhead).

x-ray films and thoracic CT showed there were irregular diffuse reticular changes $(\mathrm{N}=4)$ or ground-glass opacities with consolidation $(\mathrm{N}=3)$ in seven patients who had respiratory symptom(s) (18.9\%) (Figure 2A and Figure 2B).

We performed RT-PCR tests after hospitalization in all patients. In 14 patients, RT-PCR tests were negative. Because chest CT has a high sensitivity with confirming lung involvement of COVID-19, we performed this prior to intervention in hemodynamically stable patients. In patients with unstable vital signs, including hypotension, dyspnea, and severe chest pain, chest CT was performed after patients' stabilization or revascularization. Thoracic CT demonstrated that there was interlobular septal and pleural thickening with patchy bronchiectasis of the lower lobes bilaterally $(\mathrm{N}=9)$ and pulmonary congestion with ground-glass opacities $(\mathrm{N}=3)$ in patients with negative RT-PCR tests (Figure 3 ).

Patients with confirmed severe CAD had higher levels of c-Trop-I, when compared with the STEMI patients with normal coronary arteries (112 ng/ml versus $13.5 \mathrm{ng} / \mathrm{ml}$ ) $(P=.001)$. Blood d-dimer and CRP levels in CAD patients and in those with normal coronary arteries were (550 versus 117) $(P=.001)$ and $(56 \mathrm{mg} / \mathrm{L}$ versus $29 \mathrm{mg} / \mathrm{lL})(P=.0034)$, respectively. Patients without $\mathrm{CAD}$ had significantly lower lymphocyte counts than patients with CAD $(P=.01)$. ALT, AST, and fibrinogen levels were similar in patients with or without CAD. During PCI, all patients received unfractionated heparin in a loading dose of $100 \mathrm{U} / \mathrm{kg}$, according to the activated clotting time (ACT) maintained $>250-300$ s. According to the guidelines of rescue PCI procedures in STEMI patients with unstable hemodynamic conditions, we administered Glyco-protein (GP) IIb/IIIa inhibitors.

The time between chest pain to reperfusion was $15.2 \pm 5.4$ hours (in general, within 12 hours). We performed CABG in four patients because of failing PCI. We continued medical treatment, including aspirin (at a daily dose of $100 \mathrm{mg}$ ), high dose intravenous prednisolone (at a daily dose of 250 $\mathrm{mg}$ ), favipiravir, and antibiotics in 12 patients who had normal coronary arteries with STEMI. For confirmation of the left ventricular functions, we performed TTE. Six patients after PCI $(20.8 \%)$ and one patient after CABG died. The reasons for mortality after PCI were sudden cardiac arrest $(\mathrm{N}=2)$, $\operatorname{ARDS}(\mathrm{N}=3)$, and a major adverse cardiocerebrovascular 


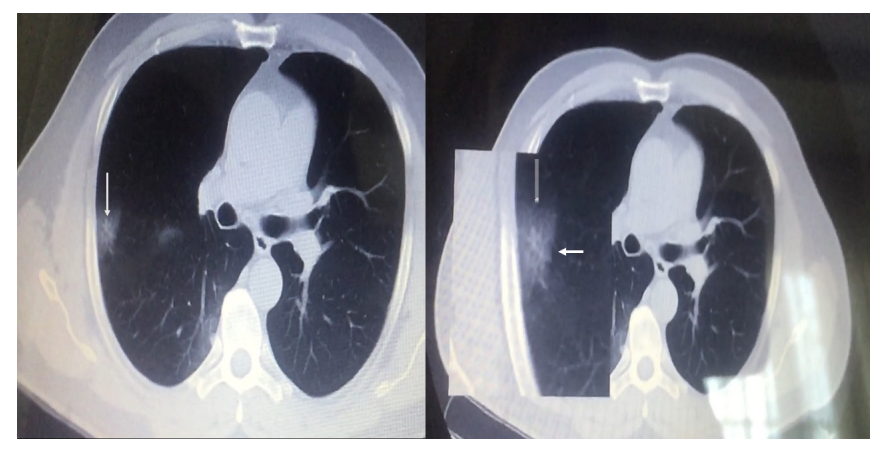

Figure 5. Example of thoracic computed tomography finding of COVID-19 infection (atypical) in a 51-year-old male with dry cough after $\mathrm{PCl}$. Axial image shows a small focal and central ground-glass opacity in the right upper lobe (white arrowhead).

event (MACCE) $(\mathrm{N}=1)$. The reason for mortality in the CABG patient was ARDS on the third postoperative day. We urgently intubated two patients after PCI because of pulmonary edema 1-3 days after coronary intervention.

All chest CTs were evaluated by blinded radiologists. New ground-glass opacities, in combination with pulmonary consolidations, were seen in three patients (12.5\%) (Figure 4). Thoracic CT findings also showed interlobular septal and pleural thickening with patchy bronchiectasis in the bilateral lower lobes. There were other pathologies in these patients. PT and aPTT were slightly increased in eight patients (21.6\%), but fibrin degradation products did not significantly change. The intubation time was longer than 48 hours in two patients (74 hours and 98 hours) because of severe hypoxia, owing to pulmonary edema and bilateral pulmonary involvement by virus after PCI. Intraaortic balloon counter pulsation was inserted, and an inotropic agent was started. Transient hemodialyses were performed in three patients because of transient renal failure. Thoracic computed tomography findings in a 51-year-old male with dry cough after PCI showed an atypical COVID-19 infection. An axial image shows a small focal and central ground-glass opacity in the patient's right-upper lobe (Figure 5).

We electively performed CABG in four patients using ECC. Respiratory symptoms, such as dyspnea and breathing difficulty, developed three days after surgery in a 47 -yearold male patient. Chest $\mathrm{x}$-ray exhibited pneumonia (Figure 6A). The first and second RT-PCR tests of this patient were negative. Axial sections of thoracic CT showed bilateral pulmonary involvement of COVID-19 in the middle and lower lobes (Figure 6B). He was hemodynamically stable. Blood levels of white blood cells increased, and lymphocytes decreased. Blood d-dimer, INR, and fibrinogen levels increased more than four times. TTE showed severely depressed left ventricular function $(\mathrm{LVEF}<30)$. Despite the use of high pressure oxygen that produced continous positive airway pressure, blood oxygen saturation decreased from $97 \%$ to $75-80 \%$. We started an inodilator and inserted an intraaortic balloon counter pulsation to support the left ventricle. We administered antibiotics, favicovir, and a high dose of intravenous steroids. We provided hemodynamic stability.

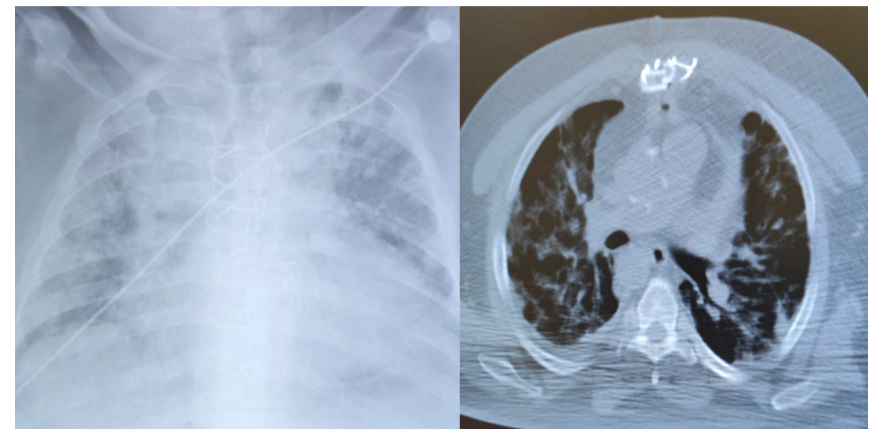

Figure 6. Postoperative chest $x$-ray demonstrating bilateral pulmonary consolidation after coronary artery bypass grafting. Thoracic computed tomography shows reticular and patchy opacities with pulmonary consolidation and pleural thickening.

Pneumonic infiltration regressed, and blood oxygen levels in air room increased to $90 \%$. Control TTE demonstrated that LVEF increased significantly (LVEF >45). He discharged home on the 29th postoperative day.

\section{DISCUSSION}

Since the beginning of the SARS-CoV-2 pandemic, there has been no common consensus regarding the treatment algorithms in confirmed COVID-19 patients with STEMI [Bangalore 2020; Giulio 2020; Shi 2020; Huang 2020; Kariyanna 2020; Singh 2020]. There are limited reports of patients with confirmed COVID-19 presenting with type-I myocardial infarction leading to STEMI who underwent PCI. Recent studies demonstrated only 10 of 18 patients with STEMI having CAD by Bangalore et al [Bangalore 2020].

Our CAD patients had peak troponin levels, CK-MB, and the severity of ventricular dysfunction seemed to be poor prognostic factors. However, we need a randomized clinical trial to clear mortality risk factors in a large number of patients. The management of COVID-19 patients with STEMI alone or those with severe electrocardiographic abnormalities who had COVID-19 presented many problems to cardiologists. According to our clinical results, we would like to emphasize that normal coronary arteries are high in patients, though patients with classical chest pain with COVID-19 were admitted to the hospital with STEMI. Our study also showed it is important to confirm COVID-19, and RT-PCR test should be done in patients with STEMI and high cardiac enzymes release. If presented with a negative test result, we should perform thoracic CT to confirm pulmonary involvement in patients with or without CAD. Myocardial enzyme elevation may be present in $28 \%$ of patients with confirmed COVID-19 because of myocardial injury without myocardial infarction [Zhou 2020; 18; AlGhatrif 2020; Choudry 2020]. If there is evidence of myocardial hypo- or akinesia Takotsubo syndrome (broken heart syndrome), treatment should be investigated using algorithm protocols.

The authors described Takotsubo syndrome in rare COVID-19 patients [Kariyanna 2020; Singh 2020; Bangalore 
2020; Dabbagh 2020; Meyer 2020]. The first case of an apical variant of Takotsubo cardiomyopathy because of COVID19 recently was reported by Kariyanna et al, as a new entity [Kariyanna 2020]. The first review of 12 confirmed COVID19 patients with Takotsubo cardiomyopathy was reported by Singh et al [Singh 2020]. Singh and other authors have hypothesized Takotsubo, due to the emotional stress by cytokine storm during COVID-19 infection [Bangalore 2020; Dabbagh 2020]. Ventriculography or TTE showed the apical ballooning in most patients with Takotsubo syndrome [Meyer 2020]. An apical hypokinesia with or without basal, mid and/ or basal segment hypokinesia are characteristic findings of Takotsubo variants [Dabbagh 2020; Meyer 2020]. Most of these patients recovered and were discharged. However, the authors have reported severe complications, such as cardiac tamponade, heart failure, cardiogenic shock, myocarditis, hypertensive crisis, or respiratory failure, in more than half of patients. We should not forget that we may encounter unusual situations with COVID-19, according to previous publications. Because three patients with normal coronary arteries had transient hypokinesia or akinesia as a result of myocarditis, we decided on atypical Takotsubo cardiomyopathy. These patients had an apical akinesia/hypokinesia with ST-segment elevation and high myocardial enzyme release. All evidence showed broken heart syndrome or Takotsubo cardiomyopathy. We treated using an inodilator, high dose steroid administration, diuretic, angiotensin-converting enzyme inhibitors, and beta-blockers. To prevent the risk of thromboembolism, we also administered a heparin drip. The myocardial contractility of the apex improved after medical treatment, and the patients were discharged from the hospital, except one young female patient. This patient developed MACCE during ICU follow up, and she died.

In the presence of normal coronary angiography, myocardial injury resulting from COVID-19 should be decided in patients with ST-segment elevation and chest pain in combination with high levels of cardiac enzyme release [AlGhatrif 2020; Choudry 2020]. Echocardiography, measurement of blood cytokine levels, levels of brain natriuretic peptide, interleukin family members, and tumor necrosis alpha may support myocarditis in these patients with confirmed COVID19. In addition, for COVID-19 patients with chest pain and STEMI who present with normal coronary angiography at hospital admission, unexplained sinusal bradycardia and/ or tachycardia and long-QT may be evidence of myocardial injury. In our series with normal coronary arteries, multifocal ST-segment changes and non-specific $\mathrm{P}$ or T-waves were evidence of myocardial involvement. We saw that sudden cardiac arrest, resulting from atrioventricular block and ventricular fibrillation, could suggest cardiac involvement by COVID19. In these situations, chest $\mathrm{x}$-ray or thoracic CT might help detect pulmonary consolidation and/or edema as a result of pneumonia. To confirm pulmonary involvement, we propose the use of thoracic CT in patients with suspected myocarditis with ischemia.

Previous studies showed that viral myocarditis frequently affected the inferolateral wall and could increase ventricular wall thickness and intracoronary microthrombi formation
[AlGhatrif 2020; Choudry 2020; Xuan 2020; World Health Organization 2005; Liu 2020; Turner 2004]. According to American College of Cardiology guidelines and previous studies in a limited number of patients, we prepared our algorithm. We divided the patients into two groups, according to hemodynamic stability or instability. We investigated regional wall motion abnormalities of the ventricle, which is evidence of a significant myocardial injury using TTE in confirmed COVID-19 patients. We monitored cardiac symptoms in combination with ST-segment changes, levels of c-TnI, and CK-MB. We immediately started medication for myocarditis using diuretics, ACE inhibitors, aspirin, beta blockers, and anticoagulant agents in the ICU. We performed coronary angiography within 1-6 hours. Coronary angiography is essential in combination with serial cardiac enzyme release to determine whether the myocardial injury reflected by the elevated enzymes levels is acute [Xuan 2020; World Health Organization 2005; Liu 2020; Turner 2004; Mallapaty 2020; Chen 2020; Poon 2020; Vaduganathan 2020; Sommerstein 2020; Madjid 2020]. Since COVID-19 has some properties that can cause myocardial injury itself and may mimic myocardial infarction, the diagnosis and treatment of CAD in patients with confirmed COVID19 admitted to hospital with STEMI carries many complex situations [Clerkin 2020; Driggin 2020; Zheng 2020]. COVID-19 might be the reason of severe arrhythmia, acute coronary syndrome, microthrombotic events, and thromboembolism [Chan 2020; Wang 2020; Huang 2020]. Cardiovascular symptoms could be the first clinical manifestation of COVID-19 in rare patients referred without typical COVID-19 symptoms, such as dysnea, fever, or dry cough [Wang 2020; Huang 2020].

The key finding of our study is classical chest pain with STEMI in patients with confirmed COVID-19 who were admitted to hospital might not be evidence of CAD, even with increased myocardial enzyme release. It has been reported in previous publications that COVID-19 might directly cause the myocardial injury by creating endothelial damage and activating macrophages. Thus, the virus may directly affect endothelial layers of coronary microvasculature, including intracoronary thrombotic burden.

Zhang et al [Zhang 2020] and Vrachatis and colleagues [Vrachatis 2020] suggested a thrombolytic administration for initial treatment of COVID-19 patients with STEMI. They proposed thrombolytic administration without coronary angiography in hemodynamically stable patients to prevent COVID-19 spread in PHWs [Zhang 2020; Vrachatis 2020]. However, delaying PCI in STEMI patients with COVID-19 and administration of thrombolytics may be an appropriate treatment strategy to prevent the safety of PHWs. Unfortunately, thrombolysis alone would cause excessive risk in these patients. In addition, thrombolytic administration may provide a low success rate and has some difficulties with the prevention of in-hospital contamination. According to our experience, we do not recommend thrombolysis in the initial treatment in these patients, considering the risk-benefit ratio. We believe that coronary angiography should be performed as a gold standard for diagnostic strategy in patients with 
confirmed COVID-19 who are admitted to the hospital with STEMI combined with high cardiac enzyme release.

Our experiences demonstrated that there was a classical chest pain in our 37 COVID-19 patients with STEMI. In these patients, cardiac enzyme release was significantly high, and some of the patients had myocardial wall abnormalities. No coronary artery occlusion was detected in 12 of 37 patients, who were admitted to the hospital due to chest pain.

As a result of our investigation, COVID-19 may precipitate STEMI with or without coronary artery disorders.

Unfortunately, the incidence of acute coronary syndrome (ACS) because of COVID-19 is unknown. Thus, we need a number of scientific reports from different countries and institutions, during the pandemic period. Since patients with symptoms of coronary artery disease are afraid of going to the hospital due to the pandemic, it is not possible to give the rate of ACS due to COVID-19. In a previous series involving 18 patients with COVID-19 with ST segment elevation, five patients $(27.7 \%)$ required PCI [Bangalore 2020]. In another study, Stefanini et al reported 28 patients with COVID-19 with STEMI, and they demonstrated culprit lesion in 17 patients (67\%) who required PCI [Stefanini 2020]. Our study confirmed CAD requiring PCI in 25 patients $(67.5 \%)$ with a success rate of $84 \%$.

According to some authors, COVID-19 may trigger coronary plaque rupture, coronary artery spasm or intracoronary microthrombus formation, due to systemic inflammation or cytokine storm [Bavishi 2020]. A number of factors, such as cytokine storm, hypoxemia of cardiac myocytes, intravascular hypercoagulation and development of coronary microvascular thrombosis, diffuse endothelial injury including the heart, and inflammation causing coronary plaque rupture might be responsible for the myocardial ischemia/infarction [Bavishi 2020]. Cardiac biomarkers can be used to aid in diagnosis as well as risk stratification. In patients with elevated hs-troponin, clinical context is important and myocarditis as well as stress induced cardiomyopathy should be considered in the differential, along with type I and type II myocardial infarction. Macrophages activation is a cause of collagenase secretation that constitutes fibrous formation on intimal plaque, which may lead to rupture [Peterson 2002]. Macrophages also are a secreted tissue factor as a procoagulant that precipitate intracoronary acute thrombosis [Peterson 2002]. COVID-19 is a cause of direct endothelial injury that might increase the risk of thrombotic occlusion. A number of publications showed the patients with ACS during the COVID19 pandemic in different countries was significantly lower than during pre-COVID-19 periods, with a reported $38-40 \%$ reduction in PCI for STEMI [Guery 2013; Aguilar 2020].

We do not know the incidence of sudden cardiac arrest during the COVID-19 pandemic in our country, which was strongly associated with the cumulative incidence of COVID19 (unpublished data). We observed sudden cardiac arrest in five adult patients (two patients were young, healthy postpregnant females) who received COVID-19 treatment 15-21 days after being discharged from the hospital. Because we cannot perform autopsies of these patients, we cannot provide any details regarding mortality.
Our series and previous reports clearly showed that the presence of myocardial injury, owing to COVID-19 itself or infarction resulting from coronary occlusion, seems to be significantly worse in COVID-19 patients. In the initial report of Anupama et al, they proposed that $12 \%$ of 41 COVID-19 patients had myocardial injury with elevated levels of cardiac biomarkers [Anupama 2019]. In a previous cohort study that involved 191 patients with COVID-19, 33 patients had acute cardiac injury, and 32 (96\%) died [Thakkar 2020]. In accordance with previous reports [Bangalore 2020; Giulio 2020], our case series of patients with STEMI and confirmed COVID-19 showed there were some variability of symptoms, such as ACS with or without respiratory symptoms. In another study of 416 patients with COVID-19, 20\% had evidence of myocardial injury, which was associated with an 11-fold increase in mortality [Singh 2020]. Thus, cardiac injury is accepted as an independent risk factor of mortality. In our patients with pre-existing coronary artery disorders with elevated myocardial enzyme levels, the mortality rate was nearly double. Our study exhibited that myocardial enzymes might be evidence of myocardial injury and predictive of the risk of in-hospital mortality. We think that patients with myocardial wall abnormalities concomitant with STEMI who have normal coronary arteries may provide evidence of typical myocarditis. We have seen that the mortality rate was similar, when we compared the patients who underwent PCI in our series. Therefore, we should clarify these results using randomized controlled clinical trials in a large case series.

We observed that PCI patients have poor clinical prognosis in our case series, especially those patients with peak myocardial enzyme release. Early stent restenosis which requires urgent re-intervention, patients with severe ventricular wall abnormality and who had some risk factors such as preexisting $\mathrm{CAD}$, and bilateral pulmonary involvement seem to be the main factors of poor outcomes. In addition, they had higher myocardial enzyme levels (more than four times the normal level or more). We think COVID-19 may continue destruction of the myocardium itself because of cytokine storm and elevated inflammatory mediators, which precipitates microvascular thrombus. Previous reports support our hypothesis [Zhou 2020; Guo 2020; AlGhatrif 2020].

Although a 14-day quarantine is recommended to prevent the spread of COVID-19 to healthy people, including PHWs during the pandemic period, there may be an increase in deaths due to myocardial infarction in those with severe CAD with hypertension and/or diabetes mellitus [Turner 2004; Mallapaty 2020; Chen 2020]. According to our experience, we have to discuss the evidence-based, risk-benefits ratio when deciding whether to postpone or proceed with PCI in suspected or confirmed CAD in COVID-19 patients. With the increase of scientific studies, many controversial issues will be revealed in the future. Understanding the clear interaction between COVID-19 and cardiovascular disease may help cardiologists with decision-making. The American College of Cardiology and American Society of Anesthesiologists suggest postponing elective PCI because they may increase the mortality risk via nasocomial infection in health care workers. 
Our case series showed that SARS-CoV-2 may trigger myocardial infarction or ischemia and may be cause of cardiomyopathy, including Takotsubo syndrome. According to our case series, urgent PCI seems to be gold standard for life saving in CAD patients with confirmed COVID-19. If there is a culprit lesion in another coronary artery - a non-infarctrelated artery - surgery may be postponed to minimize nasocomial spread of COVID-19. This would also shorten hospitalization. The cardiac team may decide a staged procedure after rescue PCI. ARDS, pneumonia, sepsis, or myocarditis and myocardial infarction, resulting from cardiac involvement by COVID-19, may be reasons for complications while waiting for PCI in patients with left ventricular dysfunction. Previous experience showed that myocardial injury can be peresent in $20 \%$ of COVID-19 patients, and it was associated with an 11-fold increase in mortality [Thakkar 2020].

Fang et al [Fang 2020] and Ai et al [Ai 2020] suggested that chest CT might be considered a primary tool for COVID-19 detection in epidemic areas. Therefore, we performed chest CT in all patients to investigate pulmonary involvement. According to previous reports, findings of chest CT showed ground-glass opacity with or without pulmonary consolidations in our patients.

\section{CONCLUSION}

Doctors should keep in mind that STEMI with chest pain, as a first sign and symptom, may be seen in COVID-19 positive patients. COVID-19 patients may be admitted to hospital with classical chest pain and STEMI with or without CAD. Since it is not clear when the pandemic will end, doctors should perform PCI in patients who are in unstable clinical condition and who require revascularization, while also being COVID positive. Because a number of CAD patients also have comorbid features, such as diabetes and/or hypertension, the risk of COVID-19 may increase mortality during the pandemic period. Fang et al suggested that the sensitivity of thoracic CT was greater than that of RT-PCR $(98 \%$ versus $71 \%, P<.001$ ) [Fang 2020]. Because of the second RT-PCR tests of four symptomatic patients were negative, we performed thoracic CT to detect whether there was pulmonary involvement. All had different degrees of bilateral pulmonary involvement. Therefore, we propose the use of thoracic CT for pulmonary involvement screening in these patients, particularly when they're exhibiting symptoms and RT-PCR testing comes back negative at hospital admission.

\section{REFERENCES}

Aguilar JB, Faust JS, Westafer LM, et al. 2020. Investigating the impact of asymptomatic carriers on COVID-19 transmission. 1;379-94.

Ai T, Yang Z, Hou H, et al. 2020. Correlation of Chest CT and RT-PCR Testing for Coronavirus Disease 2019 (COVID-19) in China: A Report of 1014 Cases. Radiology. 296(2):32-40.

AlGhatrif M, Cingolani O, Lakatta EG. 2020. The dilemma of coronavirus disease 2019, aging, and cardiovascular disease: Insights from cardiovascular aging science. JAMA Cardiology. 747-48.

Anupama BK, Chaudhuri D. 2019. A Review of Acute Myocardial Injury in Coronavirus Disease 2019. Cureus. 12(6): e8426.

Bangalore S, Atul S, Slotwiner A. 2020. ST-segment elevation in patients with COVID-19-a case series. NEJM. 382:2478-80.

Bangalore S, Sharma A, Slotwiner A, Yatskar L, Harari R, Shah B, Ibrahim H, Friedman GH, Thompson C, Alviar CL, Chadow HL, Fishman GI, Reynolds HR, Keller N, Hochman JS. 2020. ST-Segment Elevation in Patients with Covid-19-A. Case Series. N Engl J Med. 17, 382:2478-80.

Bavishi C, Bonow RO, Trivedi V, Abbott JD, Messerli FH, Bhattd DL. 2020. Acute myocardial injury in patients hospitalized with COVID-19 infection: A review. Prog Cardiovasc Dis. 63(5): 682-89.

Chan JF, Yuan S, Kok KH, et al. 2020. A familial cluster of pneumonia associated with the 2019 novel coronavirus indicating person-to-person transmission: a study of a family cluster. Lancet. 395:514-23.

Chen N, Zhou M, Dong X, et al. 2020. Epidemiological and clinical characteristics of 99 cases of 2019 novel coronavirus pneumonia in Wuhan, China: a descriptive study. Lancet. 15;395:507-13.

Chieffo A, Stefanini GG, Price S, et al. 2020. EAPCI position statement on invasive management of acute coronary syndromes during the COVID-19 pandemic. Eur Heart J. 41:1839-851.

Choudry FA, Hamshere SM, Rathod KS, Akhtar MM, Archbold RA, Guttmann OP, et al. 2020. High Thrombus Burden in Patients with COVID-19 Presenting with ST-Elevation Myocardial Infarction. Journal of the American College of Cardiology.

Clerkin KJ, et al. 2020. COVID-19 and cardiovascular disease. Circulation. 41:1648-1655.

Curzen N. 2020. An extended statement by the British Cardiovascular Intervention Society president regarding the COVID-19 pandemic. Interv Cardiol Rev. 15:01.

Dabbagh MF, Aurora L, D’Souza P, Weinmann AJ, Bhargava P, Basir MB. 2020. Cardiac tamponade secondary to COVID-19. JACC Case Rep. 2:1326-330.

Driggin E, et al. 2020. Cardiovascular considerations for patients, health care workers, and health systems during the COVID-19 pandemic. J Am Coll Cardiol. 75;2352-2371.

Fang Y, Zhang H, Xie J, et al. 2020. Sensitivity of chest CT for COVID19: comparison to RT-PCR. Radiol. 19:432.

Giulio G. Montorfano SM, Trabattoni D, et al. 2020. ST-Elevation Myocardial Infarction in Patients with COVID-19: Clinical and Angiographic Outcomes. Circulation. 141:2113-16.

Guery B, Poissy J, El Mansouf L, et al. 2013. Clinical features and viral diagnosis of two cases of infection with Middle East Respiratory Syndrome coronavirus: a report of nosocomial transmission. Lancet. 29;381:2265-72.

Guo T, Fan Y, Chen M, Wu X, Zhang L, He T, et al. 2020. Cardiovascular Implications of Fatal Outcomes of Patients With Coronavirus Disease 2019 (COVID-19). JAMA Cardiol. 5(7):811-18.

Huang C, Wang Y, Li X, et al. 2020. Clinical features of patients infected with 2019 novel coronavirus in Wuhan, China. Lancet. 15;395:497-506.

Ing ZC, Zhu HD, Yan XW, Chai WZ, Zhang S. 2020. Recommendations from the Peking Union Medical College Hospital for the management of acute myocardial infarction during the COVID-19 out break. Eur Heart J. 41:1791-94. 
Kariyanna PT, Chandrakumar HP, Jayarangaiah A, Khan A, Vulkanov V, Ashamalla M, et al. 2020. Apical Takotsubo Cardiomyopathy in a COVID-19 Patient Presenting with Stroke: A Case Report and Pathophysiologic Insights. Am J Med Case Rep. 8:350-357.

Liu Y, Gayle AA, Wilder-Smith A, et al. 2020. The reproductive number of COVID-19 is higher compared to SARS coronavirus. J Trav Med. $13 ; 27(2) .21$.

Madjid M, Safavi-Naeini P, Solomon SD, Vardeny O. 2020. Potential effects of coronaviruses on the cardiovascular system: a review. JAMA Cardiol. 5:831-40.

Mallapaty S. 2020. Why does the coronavirus spread so easily between people? Nature. 579:183.

Meyer P, Degrauwe S, Van Delden C, Ghadri JR, Templin C. 2020. Typical takotsubo syndrome triggered by SARS-CoV-2 infection. Eur Heart J. 41:1860.

Peterson ED, Coombs LP, Ferguson TB, et al. 2002. STS national cardiac data base investigators. Hospital variability in length of stay after coronary artery bypass surgery: results from the society of thoracic surgeon's national cardiac database. Ann Thorac Surg. 1;74:464-73.

Poon LL, Peiris M. 2020. Emergence of a novel human corona virus threatening human health. Nature Medicine. Feb 27:1e2.

Sala S, Peretto G, Gramegna M, Palmisano A, Villatore A, Vignale D, et al. 2020. Acute myocarditis presenting as a reverse Takotsubo syndrome in a patient with SARS-CoV-2 respiratory infection. Eur Heart J. 41:1861-62.

Shi S. Kin B, S Bo. 2020. Association of cardiac injury with mortality in hospitalized patients with COVID-19 in Wuhan, China. JAMA Cardiol. JAMA Cardiol. 5(7):802-810.

Singh S, Desai R, Gandhi Z, Fong HK, Doreswamy S, Desai V, Chockalingam A, et al. 2020. Takotsubo Syndrome in Patients with COVID-19: a Systematic Review of Published Cases. SN Compr Clin Med. 6:1-7.

Sommerstein R, Kochen MM, Messerli FH, Gräni C. 2020. Coronavirus disease 2019 (COVID-19): Do angiotensin-converting enzyme inhibitors/angiotensin receptor blockers have a biphasic effect? J Am Heart Assoc. 9(7):e016509.

Stefanini G, Montorfano M, Trabattoni D, Andreini D, Ferrante G, et al. 2020. ST-elevation myocardial infarction in patients with COVID-19: clinical and angiographic outcomes. Circulation. 141:2113-16.

Thakkar S, Arora S, Kumar A, Jaswaney R, Faisaluddin M, Ud Din MA,
Shariff M, Barssoum K. 2020. A Systematic Review of the Cardiovascular Manifestations and Outcomes in the Setting of Coronavirus-19 Disease. Clin Med Insights Cardiol. 14:1179546820977196.

Turner AJ, Hiscox JA, Hooper NM. 2004. ACE2: from vasopeptidaseto SARS virus receptor. Trends in Pharmacological Sciences. 1;25:2910-14.

Vaduganathan M, Vardeny O, Michel T, McMurray JJV, Pfeffer MA, Solomon SD. 2020. Renin-angiotensin-aldosterone system inhibitors in patients with Covid-19. NEJM. 382(17): 1653-59.

Vrachatis DA, Deftereos S, Stefanini GG. 2020. STEMI in COVID-19 patients: thrombolysis-first approach could yield more risk than benefit. Euro Heart J. 4141-42.

Wang D, Hu B, Hu C, et al. 2020. Clinical characteristics of 138 hospitalized patients with 2019 novel coronaviruse infected pneumonia in Wuhan, China. JAMA. 17;323:1061-9.

Welt FGP, Shah PB, Aronow HD, Bortnick AE, Henry TD, Sherwood MW, Young MN, Davidson LJ, Kadavath S, Mahmud E, et al. 2020. American College of Cardiology's Interventional Council and the Society for Cardiovascular Angiography and Interventions. Catheterization laboratory considerations during the coronavirus (COVID-19) pandemic: from the ACC's Interventional Council and SCAI. J Am Coll Cardiol. $75: 2372-2375$.

World Health Organization. 2005. Statement on the second meeting of the International Health Regulations Emergency Committee regarding the outbreak of novel coronavirus (2019-nCoV). Geneva, Switzerland.

World Health Organization. 2019. Coronavirus disease (COVID19) Pandemic. https://www.who.int/emergencies/diseases/ novel-coronavirus-2019.

Xuan TM, Wang XX, Pu XY, Han WL, Guo XG. 2020. Primary Percutaneous Coronary Intervention in a COVID-19 Patient with STsegment Elevation Myocardial Infarction after Lung Transplantation: A Case Report. J Zhejiang Univ Sci B. 21:411-415.

Zhang L, Fan Y, Lu Z. 2020. Experiences and lesson strategies for cardiology from the COVID-19 outbreak in Wuhan, China, by 'on the scene' cardiologists. Eur Heart J. Apr 3: ehaa266.

Zheng YY, Ma YT, Zhang JY, Xie X. 2020. Covid-19 and the cardiovascular system. Nat Rev Cardiol. 17;259-260.

Zhou F, Yu T, Du R, Fan G, Liu Y, Liu Z, et al. 2020. Clinical course and risk factors for mortality of adult inpatients with COVID-19 in Wuhan, China: A retrospective cohort study. Lancet. 395:1054-62. 